EDITORIAL

\title{
Our criteria for publication
}

This journal champions an efficient and rigorous peer review process. As part of our continued commitment to ensuring that editorial decisions are made consistently and fairly, and are easily understood, we have announced a refreshed set of criteria for publication against which all papers submitted to the journal will be assessed. This change is made in a spirit of providing further transparency and clarity in relation to our editorial decision-making.

\section{The role of peer review}

$t$ is widely accepted that peer review is the most valid form of scholarly evaluation and, as such, is a cornerstone of the process of bringing academic research to publication. A rigorous and fair peer review system should therefore be the backbone of any reputable academic publication. That may seem like a truism, but given that journals come increasingly in different shapes and sizes, it is worth repeating.

At this journal we place strong emphasis on providing authors with an efficient and robust peer review experience-one that is underpinned by high ethical and editorial standards. As journal editors our role, at its best, should be to guide authors in developing and strengthening their work to the point where it is acceptable for publication. We are committed to ensuring that our peer review process is a collaborative one between authors, editors and reviewers. Ultimately, peer review should be both constructive and instructive.

\section{Our scope and vision}

This journal is broad in scope and is committed to publishing research arising in all fields within the humanities, social and behavioural sciences. We additionally actively welcome interdisciplinary perspectives, and we are particularly proud that the journal has also become established as an attractive outlet for those whose research may not find a natural home in niche or single discipline-focused titles. The body of research papers we have published since we began publication in 2015 strongly reflects this ethos.

Providing a quality service to readers, reviewers and authors is a key priority for us. Central to this is ensuring that our peer review process is appropriate for the full breadth of academic communities we seek to serve-from economics and linguistics, social psychology and archaeology, through to medical humanities and quantitative sociology. This, for instance, involves a recognition that research methods, practices and conceptual frameworks can differ significantly from discipline to discipline; as do expectations and perceptions of what constitutes 'novelty', 'originality' and 'impact'. Not to mention that such challenges can be further amplified in the context of assessing interdisciplinary research.

Against this backdrop we have developed a refreshed list of 'criteria for publication' for our editors and reviewers to use as a framework when assessing submitted manuscripts. Fundamentally, our intention is to provide further transparency and clarity in regards to editorial decisionmaking at the journal.

\section{Criteria for publication}

To be publishable in this journal a paper should satisfy all of the following criteria:

1. Report research that is within the journal's scope;

2. Pose a clear and valid research question;

3. Be academically sound in methodology and analysis;

4. Provide appropriate evidence or reasoning for the conclusions;

5. Make a contribution to the literature (we do not consider abstracts and internet preprints

to compromise this);

6. Be presented in an intelligible fashion and in standard English. 
Underpinning these criteria are four core intentions: first, to ensure that editorial decisions are made fairly and consistently and, importantly, are easily understood by authors; second, to continue to offer a broad and inclusive outlet for rigorous research, including interdisciplinary work; third, to recognise that perceptions of concepts like 'significance', 'novelty' and 'impact' can vary between the disparate disciplines we serve; and fourth, to acknowledge that such perceptions often only become fully apparent and quantified post-publication.

Several observations are warranted. First, naturally, our criteria for publication stipulate that, to be publishable, a paper should be considered fully methodologically sound (no. 3). We of course recognise that precise expectations in this respect may vary by discipline and research type, and our editors, as experienced academics, will be mindful of these when handling papers. For instance, crudely, in quantitative work any statistical tests or data analysis must be judged valid and reproducible; while in qualitative studies, there must be a clear and logical coherence to any arguments developed. Second, we expect a paper to report research that makes a contribution to the literatureirrespective to any perceived magnitude or defined degree of advance (no. 5). That is to say, a paper should report something specific and not simply repeat what is already known in the literature. This ensures the journal is inclusive to studies reporting incremental advances, those presenting negative results, as well as replication studies (if academically justified).

Our editors have an important responsibility to provide a neutral bridge between referees and authors. This is particularly important in instances where consensus is lacking between reviewers' reports. Our editors will ensure that judgements over suitability for publication ('accept/revise, vs. reject') are based solely on our six core criteria. Judgements of perceived impact, degree of advance, or significance will not inform those decisions.

To conclude, the clarifications outlined here are inextricably linked to this journal's guiding philosophy: to facilitate the publication of research that enriches the body of knowledge in all disciplines in-and between - the humanities, behavioural and social sciences.

Published online: 13 January 2020

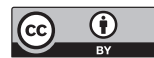

Open Access This article is licensed under a Creative Commons Attribution 4.0 International License, which permits use, sharing, adaptation, distribution and reproduction in any medium or format, as long as you give appropriate credit to the original author(s) and the source, provide a link to the Creative Commons license, and indicate if changes were made. The images or other third party material in this article are included in the article's Creative Commons license, unless indicated otherwise in a credit line to the material. If material is not included in the article's Creative Commons license and your intended use is not permitted by statutory regulation or exceeds the permitted use, you will need to obtain permission directly from the copyright holder. To view a copy of this license, visit http:// creativecommons.org/licenses/by/4.0/.

(C) Springer Nature Limited 2020 